\title{
Wann die Hebamme ärztlichem Vorgehen widersprechen muss
}

\author{
Matthias Diefenbacher, Rechtsanwalt, Heidelberg
}

\author{
Im Kreißsaal ist die Hebamme dem Arzt untergeordnet. Den ärztlichen Anweisungen \\ bei der Geburt hat sie Folge zu leisten. Die Hierarchie endet, wenn ein Arzt regelwidrig \\ handelt und die Hebamme dies erkennt. Greift sie trotz besseren Fachwissens nicht ein, \\ riskiert sie Schadensersatzforderungen, strafrechtliche Konsequenzen und ihre Berufs- \\ erlaubnis.
}

\section{Erlaubte Tätigkeiten der Hebamme}

- In $\S 4$ HebG ist in Absatz 1 geregelt, dass zur Leistung von Geburtshilfe abgesehen von Notfällen - außer Ärztinnen und Ärzten nur Personen mit einer Erlaubnis zur Führung der Berufsbezeichnung „Hebamme“ oder „Entbindungspfleger“ berechtigt sind.

- In $\S 4$ Absatz 2 HebG ist der Begriff der „Geburtshilfe“ definiert: „Geburtshilfe im Sinn des Absatz 1 umfasst Überwachung des Geburtsvorgangs von Beginn der Wehen an, Hilfe bei der Geburt und Überwachung des Wochenbettverlaufs." Da in dieser Definition der gesamte Bereich der Schwangerenvorsorge nicht genannt ist, ist auf $\S 5 \mathrm{HebG}$ zu verweisen, der als Ausbildungsziel insbesondere auch nennt, dass „die Ausbildung dazu befähigen soll, Frauen auch während der Schwangerschaft (...) Rat zu erteilen und die notwendige Fürsorge zu gewähren“. Im Unterschied zur Definition der Geburtshilfe in $\S 4$ Absatz 2 HebG findet sich daher in $\S 5$ HebG auch die Versorgung der Frau während der Schwangerschaft als Ausbildungsziel. Damit gehört auch die Vorsorge zu den für die Hebamme erlaubten Tätigkeiten.

- Weitere Kompetenzen der Hebamme ergeben sich aus den Landesberufsordnungen (dort abhängig vom Bundesland jeweils in $\S 1$ oder $\S 2$ ), den nahezu gleichlautenden EU-Vorschriften, den relativ neuen Vorschriften der $\S \S 24 a$ ff. SGB V und der Leistungsbeschreibung und dem Vergütungsverzeichnis zum Vertrag über Hebammenhilfe nach § 134a SGB V.

und Risikofaktoren zu achten und bei deren Auftreten die Hinzuziehung eines Arztes oder die Einweisung in ein Krankenhaus zu veranlassen. Beim Eintritt ernsthafter Komplikationen muss die Hebamme den zuständigen Arzt unterrichten. Wird die Hinzuziehung eines Arztes von Schwangeren, Gebärenden oder Wöchnerinnen gewünscht, muss auch diesem Wunsch entsprochen werden.

\section{Verhältnis zwischen Hebamme und Arzt}

Diese Grundsätze haben dann auch Auswirkungen auf das Verhältnis zwischen Hebamme und Arzt, bei Beleghebammen bzw. angestellten Hebammen auch zum Krankenhausträger.

\section{Haftung des Krankenhauses}

\section{Hinzuziehungspflicht des Arztes}

Für Ärztinnen und Ärzte gilt nach $\S 4$ HebG, dass sie (immer) „verpflichtet sind, dafür Sorge zu tragen, dass bei einer Entbindung eine Hebamme oder ein Entbindungspfleger zugezogen wird“. Um diese Hinzuziehungspflicht zu erfüllen, ist ein ernsthaftes Bemühen des Arztes verpflichtend. Dies gilt im Übrigen auch für die Hinzuziehung einer Hebamme bei einer Sectio.

Aus § 4 HebG lässt sich daher auch die Abgrenzung der Tätigkeit einer Hebamme zur ärztlichen Tätigkeit entnehmen. Die Hebamme darf regelgerechte Geburten vollständig selbst durchführen. Das Behandeln regelwidriger Vorgänge ist Ärzten vorbehalten. Deshalb haben Hebammen auch auf Regelwidrigkeiten
Eine Haftung des Krankenhauses besteht insbesondere dann, wenn den Verantwortlichen ein Organisationsverschulden nachgewiesen werden kann.

Steht z.B. für die Überwachung eines CTG nur eine Krankenschwester zur Verfügung, so haftet das Krankenhaus für ein solches Organisationsverschulden. Das Krankenhaus muss darüber hinaus dafür sorgen, dass ein Belegarzt das 
Krankenpflegepersonal (nur) mit Aufgaben betraut, die dessen Kompetenz nicht überschreiten (Urteil des BGH vom 16.04.1996). Sollte eine Hebamme nicht hinreichend mit dem CTG vertraut sein, haftet ein Belegarzt, der die Leitung der Geburt übernommen hat, auch für Fehler der Hebamme (Urteil des OLG Zelle vom 28.07.1997). In diesem Zusammenhang muss die Hebamme bei einem hoch pathologischen CTG einen Arzt nicht nur benachrichtigen, sondern sein Kommen auch als „dringlichst“ darstellen (Urteil des LG Darmstadt vom 21.04.1994).

Sollte ein Arzt die telefonische Anweisung erteilen, wehenfördernde Mittel einzusetzen, ohne dass die Überwachung durch ein CTG möglich ist, liegt nach der Rechtsprechung ein „grober Behandlungsfehler" vor (so auch in $\S 630 \mathrm{~h} \mathrm{Ab-}$ satz 5 BGB). In diesem Zusammenhang muss auch die Hebamme stets wissen, dass ein solches Vorgehen gegen die Regeln ärztlicher Kunst verstößt. Macht sie dem Arzt keinen entsprechenden Vorhalt, ist sie für den eingetretenen Schaden mitverantwortlich (Urteil des OLG Frankfurt vom 06.04.1990).

In diesen Fällen der Mithaftung bedeutet dies für die Hebamme, dass sie als Gesamtschuldnerin nach $\S 426$ BGB die Haftung mit übernimmt. Das Wesen der Gesamtschuld liegt insbesondere darin, dass die Gesamtschuldner im Verhältnis zueinander zu gleichen Anteilen verpflichtet sind. Kann von einem der Gesamtschuldner der auf ihn entfallende (Teil-)Beitrag nicht erlangt werden, so ist dessen Anteil von den übrigen Schuldnern zu tragen ( $\$ 426$ Abs. 1 BGB). In der Praxis bedeutet dies, dass die geschädigte Frau sich bemühen wird, möglichst viele Verantwortliche in Anspruch zu nehmen, um eine größtmögliche Sicherheit zu erlangen, mindestens einen leistungsfähigen Schuldner verklagen zu können. Infrage kommen hier das Krankenhaus, sämtliche Ärzte in der Hierarchie des Krankenhauses sowie sämtliche im Kreißsaal anwesenden Hebammen bis hin zur Hebammenschülerin. Sollte der schlimmste Fall eintreten, dass alle anderen Verantwortlichen nicht leistungsfähig sind, könnte die Geschädigte letztendlich auch die Hebamme alleine für die Gesamtsumme in Anspruch nehmen. Die Hebamme hätte dann (nur noch) die Möglichkeit, die auf die anderen Beteiligten entfallenden Anteile bei diesen im Innenverhältnis geltend $\mathrm{zu}$ machen. Nach außen haftet nach $\S 426$ BGB jeder Gesamtschuldner allerdings nur einmal auf die gesamte Summe, die insgesamt auch nur einmal verlangt werden kann (d.h. nicht von jedem der Beteiligten jeweils einzeln).

\section{Verhältnis zwischen Hebamme und Arzt}

Nach der Übernahme der Geburt durch den Arzt ist die Hebamme ausschließlich dessen Erfüllungsgehilfin ( $§ 278$ BGB). Dies gilt auch im Verhältnis zwischen dem Belegarzt und der im Krankenhaus angestellten Hebamme. Die Hebamme unterliegt dann dem Weisungs- und Direktionsrecht des Belegarztes (Urteil des OLG Karlsruhe vom 13.10.2004). Das Krankenhaus haftet dann nicht, wenn die Hebamme nur Erfüllungsgehilfin des Belegarztes ist, da das Krankenhaus die Leistungen von Belegarzt und Beleghebamme nicht schuldet (Urteil des OLG Koblenz vom 26.07.2000 und des BGH vom 07.12.2004). Der BGH hat in einem Urteil vom 14.02.1995 klargestellt, dass der Belegarzt auch für Fehler der freiberuflich tätigen Hebamme verantwortlich ist, die die Geburt bei zeitweiliger Abwesenheit des Arztes überwacht. Der Krankenhausträger haftet nur für die Fehler der bei ihm angestellten Hebammen, die dem Belegarzt nicht zugerechnet werden können (Urteil des BGH vom 16.05.2000 und des OLG Karlsruhe vom 16.05.2001).

Auch ein Arzt, der anstelle des eine Geburt betreuenden Belegarztes absprachegemäß die Geburt weiterleitet, ist als Vertreter des Belegarztes dessen Erfüllungs- und Verrichtungsgehilfe, auch wenn er selbst Belegarzt des Krankenhauses ist. Die Hebamme kann auch nach 
der Übernahme der Geburtsleitung durch diesen Arzt wiederum Erfüllungs- und Verrichtungsgehilfin des (zweiten) Belegarztes sein (Urteil des OLG Stuttgart vom 19.09.2000).

Weitere Besonderheiten können sich im Rahmen der Rufbereitschaft ergeben. Für Fehler einer Hebamme muss dann der in Rufbereitschaft wartende Belegarzt erst ab dem Zeitpunkt einstehen, in welchem die Leitung der Geburt seine eigene Vertragsaufgabe geworden ist.

\section{Remonstrationspflicht}

\section{Arbeitet eine erfahrene Hebamme mit} einer unerfahrenen Ärztin zusammen, ist die Hebamme für die Fehlentscheidungen der Ärztin dann mitverantwortlich, wenn sie die Ärztin auf die Fehler nicht aufmerksam gemacht hat (Urteil des AG Demmin vom 23.07.1998).

Die unerfahrene Ärztin ihrerseits haftet für Fehler der Hebamme nur dann, wenn für sie Fehler der Hebamme erkennbar werden und sie dann keine Hilfe eines Facharztes hinzuzieht (Urteil des OLG Stuttgart vom 08.07.2003).

Allerdings ist eine Hebamme für Versäumnisse in der Endphase der Entbindung dann nicht verantwortlich, wenn ein ärztlicher Geburtshelfer anwesend ist und die Verantwortung für die Entscheidungen trägt (Urteil des OLG Düsseldorf vom 25.11.1999).

\section{Offensichtliche Fehlbehandlung}

Das ist allerdings dann nicht der Fall, wenn es sich um eine offensichtliche Fehlbehandlung handelt und eine Remonstrationspflicht der Hebamme besteht.

Die zentrale Entscheidung über eine solche Verpflichtung hat das OLG Düsseldorf am 26.04.2007 (AZ: 8 U 37/05; Hebammenforum 12/2007, S.1002) getroffen. In diesem Urteil wurde entschieden, dass

\section{Pflichten der Hebamme}

- Die Hebamme ist bei einer regelgerechten Geburt dem Arzt gleichgestellt.

- Dies ist nicht mehr der Fall, wenn der Arzt die (pathologische) Geburt leitet und die Hebamme (nur noch) seine Gehilfin ist.

- Diese Hierarchie endet jedoch, sobald die Hebamme aufgrund ihrer eigenen geburtshilflichen Ausbildung erkennen muss, dass das Vorgehen des Arztes vollkommen regelwidrig und unverständlich ist (OLG Düsseldorf, a. a. O., Rdnr. 96).

- Die Hebamme muss neben dem Arzt in eigener Verantwortung tätig werden, wenn sie erkennt, dass das Untätigbleiben des Arztes über einen längeren Zeitraum gegen alle elementaren Regeln der Geburtshilfe verstößt (OLG Düsseldorf, a. a. O., Rdnr. 107, 108). Dies kann der Fall sein, wenn der Arzt unsicher und/oder unschlüssig ist, der Arzt nur halbherzige Hinweise gibt oder durch den Arzt eine falsche Behandlung angeordnet wird. In diesen Fällen darf sich die Hebamme nicht mehr auf gutes Zureden beschränken. Es wird eine laute und drastische Intervention bis hin zum Eklat erwartet.

- Die Hebamme sollte sich in den Fällen einer Remonstration zunächst intern, d.h. nur gegenüber dem Arzt beschweren. Liegt ein eiliger Notfall vor, muss extern remonstriert werden, d.h. interne Hierarchiestrukturen helfen nicht mehr weiter und müssen verlassen werden, um z.B. selbst einzugreifen oder externe Hilfe (Notarzt) hinzuzuziehen.

eine Hebamme zwar von dem Moment an, in dem der Arzt bei der Geburt hinzutritt, dessen Gehilfin ist und seinen Anweisungen Folge zu leisten hat. Dies bedeutet indessen nicht, dass sie sich dem Arzt jederzeit und in jeder Situation völlig unterordnen muss oder darf. Die Hierarchie endet dann, wenn die Hebamme aufgrund ihrer eigenen geburtshilflichen Ausbildung erkennen muss, dass das Vorgehen des Arztes vollkommen regelwidrig und unverständlich ist. In diesem Fall darf die Hebamme nicht alles mitmachen oder zulassen, was der Arzt anordnet oder auch unterlässt. Sie muss „erkennen, dass das Untätigbleiben eines Arztes über einen längeren Zeitraum angesichts einer Notsituation des Kindes gegen alle elementaren Regeln der Geburtshilfe verstößt. Sie hat in einem solchen Fall den Arzt vehement und mit allem Nachdruck aufzufordern, die Entbindung des Kindes zu beschleunigen oder - falls der Arzt weiter untätig bleibt - selbst die Geburt (...) voranzutreiben“ (Hebammenforum 2007, 1002). „Die Hebamme darf sich bei für sie erkennbaren groben Versäumnissen des Arztes nicht hinter dessen Autorität zurückziehen und ebenfalls untätig bleiben. Sie ist wie der Arzt der Geburtshilfe verpflichtet und hat als ebenfalls verantwortliche Geburtshelferin alles zum Wohle des Kindes zu unternehmen. Greift sie in einer Notsituation nicht ein, verletzt sie in grober Weise ihre Berufspflichten.“

Ein bloßer Hinweis an den Arzt reicht je nach Schwere des Verstoßes nicht aus.

Im konkreten Fall hatte der Assistenzarzt die Schwangere zwar aufgefordert, die Geburtswanne zu verlassen, aber nicht den Eindruck vermittelt, als ob es eilig sei. Danach hätte die Hebamme die Initiative ergreifen müssen. Angesichts der Notsituation im konkreten Fall hätte sie die Schwangere notfalls lautstark und vehement veranlassen müssen, die Wanne zu verlassen. Die Verteidigung der Hebamme, die Schwangere habe sich geweigert, die Wanne zu verlassen, war nicht ausreichend. In einer Notsituation, in der für eine entsprechende Aufklärung (zum Verhalten der Schwangeren und den sich daraus ergebenden Folgen) keine Zeit mehr ist, dürfen sich Hebamme und Arzt nicht mehr auf gutes Zureden be- 
schränken. Es wird eine laute und drastische Intervention bis hin zum Eklat erwartet. All das ist sehr sorgfältig zu dokumentieren!

\section{Allgemeine Haftungsfragen}

Schreitet die Hebamme trotz besseren Fachwissens und praktischer Erfahrungen und trotz Erkennens eines Fehlverfahrens des Arztes nicht ein, gerät sie in die Gefahr einer Haftung auf Schadensersatz. Dies kann sich im Rahmen eines Behandlungsvertrags ( $§ \S 630 \mathrm{a}$ ff. BGB) aus $\S 280$ BGB ergeben. Jeder Behandlungsfehler bzw. jedes Fehlverhalten im Rahmen des Behandlungsvertrags stellt eine Pflichtverletzung im Sinn dieser Vorschrift dar.

Es kommt jedoch auch eine Haftung aus unerlaubter Handlung ( $\S 823$ BGB) infrage, wenn die Hebamme durch ihr Ver- halten mindestens fahrlässig den Körper oder die Gesundheit der Frau widerrechtlich, d.h. ohne deren Einwilligung, verletzt. Dies kann auch durch Unterlassen geschehen, da die Hebamme aufgrund des Behandlungsvertrags oder der Übernahme einer tatsächlichen Verantwortung für die Frau eine Garantenpflicht innehat.

Daneben kann auch eine strafrechtliche Haftung, insbesondere wegen Körperverletzung bestehen. Auch eine Körperverletzung ( $\S \S 223 \mathrm{ff}$. StGB) kann durch Unterlassen begangen werden.

Schließlich kann ein solches Verhalten („Schweigen“ im Kreißsaal) auch eine unterlassene Hilfeleistung darstellen (§ 323c StGB). Greift die Hebamme bei einem Unglücksfall oder einer Gefahr für die Frau nicht ein, obwohl dies erforderlich und ihr den Umständen nach zuzumuten ist, kann sie mit Freiheitsstrafe bis zu einem Jahr oder mit Geldstrafe bestraft werden. Es ist wichtig, darauf hinzuweisen, dass sich die Hebamme in diesen Fällen auch nicht damit entlasten kann, „lediglich Befehle befolgt zu haben“. Es wird erwartet, dass die Hebamme sich aufgrund ihres Fachwissens und ihrer praktischen Erfahrung im Rahmen der Remonstrationspflicht gegen solche fehlerhaften „Befehle“ wehrt und Gegenmaßnahmen ergreift. Diese können insbesondere in der Verständigung eines anderen Facharztes oder des Notarztes liegen.

\section{Berufserlaubnis}

$\mathrm{Zu}$ guter Letzt ist darauf hinzuweisen, dass es sich aus einem solchen regelwidrigen Verhalten der Hebamme ergeben kann, dass die Hebamme sich eines Verhaltens schuldig gemacht hat, aus dem sich die Unzuverlässigkeit zur Ausübung des Hebammenberufs ergibt ( $§ 3$ Abs. 2 in Verbindung mit $\S 2$ Abs. 1 Nr. 2 HebG). Sind diese Voraussetzungen erfüllt, bleibt der zuständigen Behörde gar nichts anderes übrig, als der Hebamme die Berufserlaubnis zu widerrufen. Einen Ermessensspielraum der Behörde sieht das Gesetz nicht vor. Die Verletzung der Remonstrationspflicht wirkt sich also sogar arbeitsrechtlich aus, da schwerwiegende Pflichtverletzungen im Kreißsaal auch zu einer Kündigung durch den Arbeitgeber führen können.

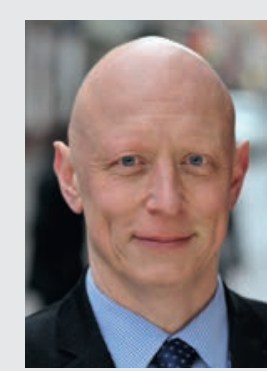

Matthias Diefenbacher

Rechtsanwalt

Vangerowstr. 2/2

69115 Heidelberg

E-Mail: Matthias@Diefenbacher.de 